\title{
Search for Muon - Electron Conversion on Gold
}

\section{W. Bert।*, F. Rosenbaum and N.M. Ryskulov}

Paul Scherrer Institute, $\mathrm{CH}$ - 5232 Villigen, Switzerland

E-mail: 'wilhelm. bertl@psi.ch

R. Engfer, E.A. Hermes, G. Kurz, A. van der Schaaf and P. Wintz

Physik-Institut der Universität Zürich, $\mathrm{CH}$ - 8057 Zürich

E-mail: andries@physik.unizh.chi

\section{J. Kuth and G. Otter}

III. Phys. Inst. B der RWTH Aachen, D - 52056 Aachen, Germany

\section{T. Kozlowski and I. Zychor}

Institute for Nuclear Studies, PL - 05-400 Otwock-Swierk, Poland

E-mail: 'kozlowski@ipj.gov.pl'

ABSTRACT: During the year 2000 the Sindrum II collaboration at the Paul-Scherrer Institute, Switzerland (PSI) performed a final run in a search for coherent $\mu^{-} \rightarrow \mathrm{e}^{-}$conversion on gold. An improvement in sensitivity by two orders of magnitude was achieved, but no candidate events for the conversion process have been seen.

\section{Introduction}

Observations on solar and atmospheric neutrinos indicate that neutrinos mix so lepton flavor would not be conserved. SINDRUM II tests lepton-flavor conservation by a search for neutrinoless $\mu^{-} \rightarrow \mathrm{e}^{-}$conversion in muonic atoms. This process and the decay $\mu \rightarrow e \gamma$ are promising signals of supersymmetric grand unification [iَ]

For $\mu^{-} \rightarrow \mathrm{e}^{-}$conversion leaving the nucleus in its ground state the transition amplitude is the coherent sum of the amplitudes for the individual nucleons which leads to an enhancement relative to the competing process of nuclear muon capture. As a result it is advantageous to choose a heavy target such as gold (see Fig. iij). Compared to lead gold has good mechanical properties allowing a lightweight self-supporting target (see Fig. (3). $)$. In addition, gold has only one stable isotope which is advantageous when searching for $\mu^{-} \rightarrow e^{+}$conversion (not subject of this talk).

In the process $\mu^{-}(A, Z) \rightarrow e^{-}(A, Z)^{g . s .}$ the electron is emitted at the kinematical endpoint for bound muon decay; in the case of gold at $95.55 \mathrm{MeV} / \mathrm{c}$.

\footnotetext{
${ }^{*}$ Speaker.
} 


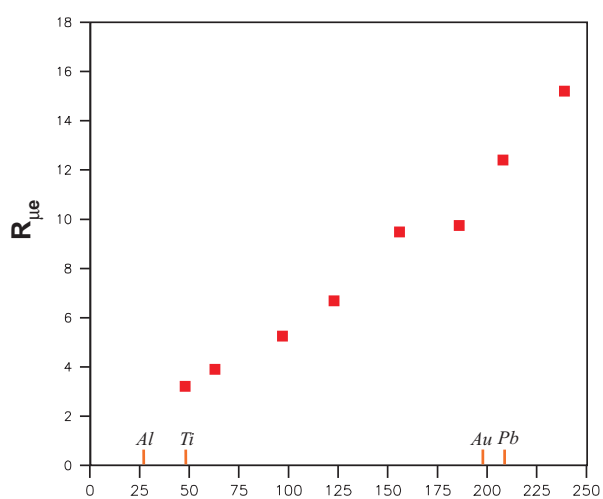

A

Figure 1: Nuclear dependence of coherent $\mu^{-} \rightarrow \mathrm{e}^{-}$conversion rate

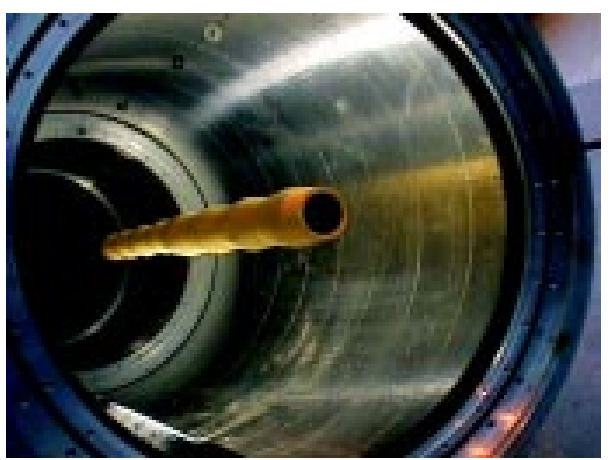

Figure 2: The hollow gold target centered in the vacuum chamber inside SINDRUM II. The self-supporting structure has a wall thickness of only $40 \mu \mathrm{m}$ and is suspended by thin Tungsten wires invisible on the picture.

\section{The SINDRUM II Spectrometer}

The SINDRUM II spectrometer consists of a superconducting solenoid, which produces a field of $1.05 \mathrm{~T}$ in a region of $1.35 \mathrm{~m}$ diameter and $1.8 \mathrm{~m}$ length, housing various cylindrical detectors. A schematic view of the spectrometer is shown in Fig. an incoming muon stopping in the gold target and the resulting decay electron. For a detailed

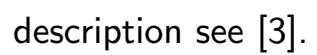

Two plastic scintillator hodoscopes and a lucite Cerenkov hodoscope are used for timing and trigger purposes. The momentum is determined from the tracks recorded in the inner drift chamber. This chamber covering the radial region $37.6-44.5 \mathrm{~cm}$ is filled with $\mathrm{CO}_{2} / \mathrm{iC}_{4} \mathrm{H}_{10}$ (70/30), a gas with a low drift velocity which results in a Lorentz deflection of $6^{\circ}$ only. The drift field is oriented radially with axial sense wires close to the outer cathode foil. This cathode is subdivided into $4.4 \mathrm{~mm}$ wide strips oriented $72^{\circ}$ relative to the sense wires. Correlated signals from sense wires and cathode strip signals arrive at the same time which allows a 3-dimensional track reconstruction. The outer radial drift chamber is filled with a $\mathrm{He} / \mathrm{iC}_{4} \mathrm{H}_{10}(88 / 12)$ mixture with large radiation length for minimal multiple scattering. No cathode readout is foreseen for this chamber.

\section{Background Sources}

The only source of intrinsic background is muon decay in orbit $\mu^{-} \mathrm{Au} \rightarrow e^{-} \nu \bar{\nu} \mathrm{Au}$. The $\mathrm{e}^{-}$ momentum distribution drops steeply toward the endpoint and the process can be suppressed sufficiently with a momentum resolution around $1 \%$.

Other sources of electrons with momenta around $95 \mathrm{MeV}$ are pions contaminating the beam and cosmic rays, mostly through intermediate photons producing asymmetric $\mathrm{e}^{+} \mathrm{e}^{-}$pairs in the target. Pions stopping in the target produce background with a probability of $\left(10^{-5}\right)$ so at most $\left(10^{4}\right)$ pions may reach the target during the $\approx 3$ months live time of the experiment. 


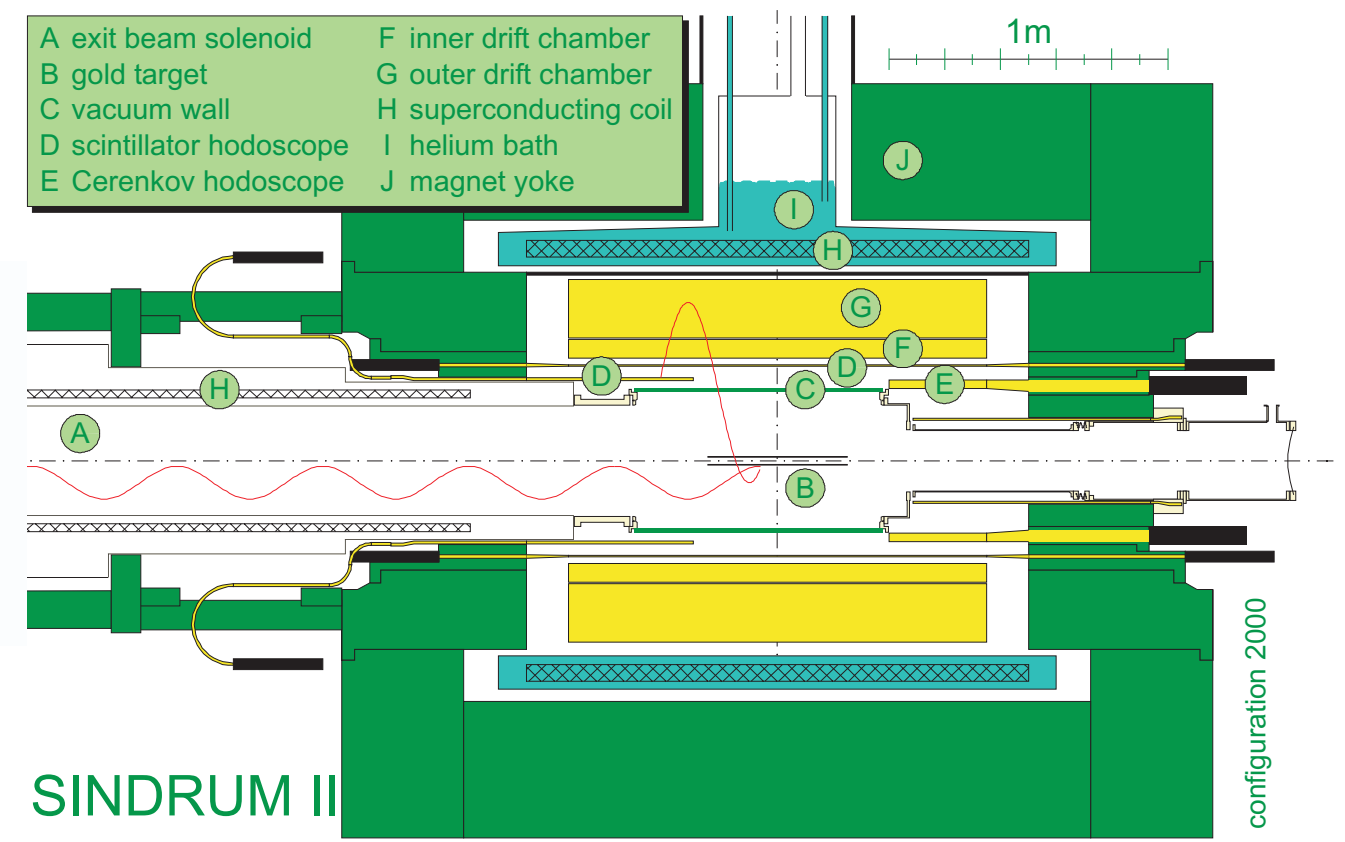

Figure 3: Cross section through SINDRUM II connected to the PMC magnet. The trajectory of a muon is shown entering from the left and stopping in the gold target. The electron trajectory originating from the decay of the resulting muonic atom is shown too. For further explanations see text.

At the selected beam momentum of $50 \mathrm{MeV} / \mathrm{c}$ the beam contains similar numbers of $\mu^{-}$and $\pi^{-}$. Pions have a mean range which is about half of the muon range. $\mathrm{A} \mathrm{CH}_{2}$ moderator of $8 \mathrm{~mm}$ thickness stops almost all pions but lets most muons pass. A $9 \mathrm{~m}$ long solenoid is used to transport the resulting broad-band muon beam to the target. The few pions that cross the degrader have very low momenta and simulation shows that $\approx 99.9 \%$ of them decay before reaching the target. Electrons from radiative pion capture in the moderator may reach the target and scatter into the detector solid angle. This background can be recognized since it is strongly peaked in forward direction and it has a characteristic time correlation with the cyclotron rf signal. Background from photons in cosmic showers is suppressed sufficiently by the spectrometer yoke. Photons may enter through the cryogenic supply tower (see Fig. 닉). This background is removed at the cost of a $5 \%$ loss in acceptance.

\section{Determination of number of muon stops}

The number of muons stopping in the target was measured during the experiment by observing the characteristic muonic gold $\mathrm{X}$-rays. Two detectors have been employed, partially in parallel: a) a large $\mathrm{Nal}(\mathrm{TI})$ crystal was used to monitor the $2 \mathrm{p}-1 \mathrm{~s}$ transitions at $5765 \mathrm{keV}$ and $5595 \mathrm{keV}$ and b) a Germanium diode was used to observe the $4 f_{5 / 2} \rightarrow 3 d_{3 / 2}$ transition at $899 \mathrm{keV}$. Both detectors were placed outside the spectrometer coil. The Germanium monitor is more accurate because of better background separation and more reliable calibration using ${ }^{137} \mathrm{Cs}$ and ${ }^{60} \mathrm{Co}$ sources. The calibration results were reproduced with an accuracy of $3 \%$ by a Monte Carlo simulation. The same code was used to determine the acceptance for gold $\mathrm{X}$-rays folded with 
the observed muon stop distribution. Given the known emission probability of the X-rays [i5] the number of muons stopping during the live time of the experiment was determined as

$$
N_{\mu}^{\text {stop }}=\left(4.30 \pm 0.3_{\text {stat }} \pm 0.3_{\text {sys }}\right) \times 10^{13} .
$$

\section{Search for $\mu^{-} \rightarrow \mathbf{e}^{-}$conversion}

The final analysis is based on the momentum spectrum of electrons originating in the target after suppression of events induced by cosmic rays. Cosmic ray events are recognized by the occurrence of additional tracks in the various detectors. The measured momentum distribution is compared with distributions from simulations of bound muon decay and coherent $\mu^{-} \rightarrow$ $\mathrm{e}^{-}$conversion in Fig. 嶰. The theoretical momentum distribution for bound muon decay was obtained from a calculation for lead [i]] corrected for the shift in the endpoint. The agreement between measured and simulated distributions is excellent, both in shape and in total number of events. No candidate events are found so the experiment results in an upper limit only.

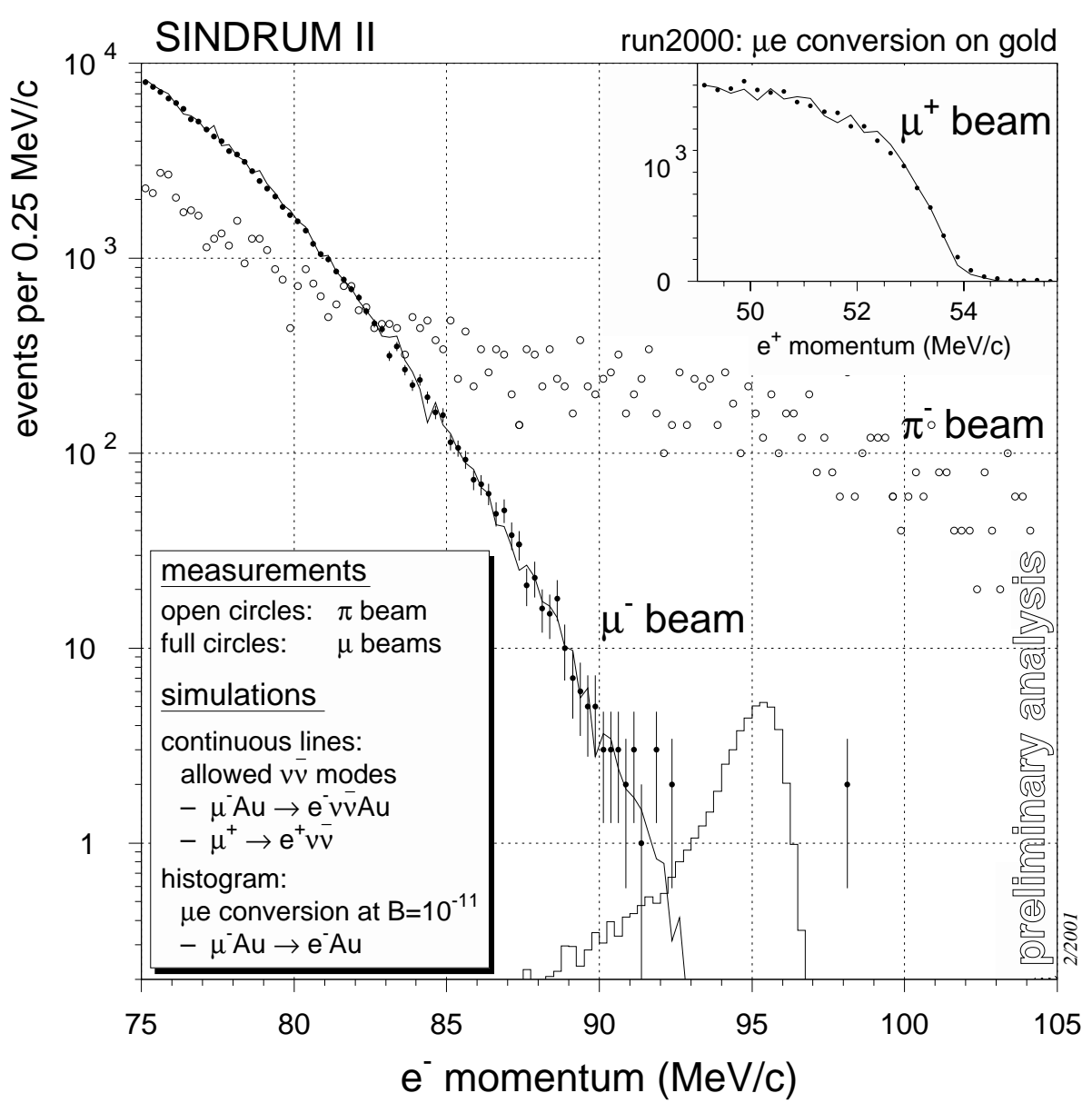

Figure 4: Momentum distributions for three different beam momenta and polarities: (i) $53 \mathrm{MeV} / \mathrm{c}$ negative, optimized for $\mu^{-}$stops, (ii) $63 \mathrm{MeV} / \mathrm{c}$ negative, optimized for $\pi^{-}$stops, and (iii) $48 \mathrm{MeV} / \mathrm{c}$ positive, for $\mu^{+}$stops. The $63 \mathrm{MeV} / \mathrm{c}$ data were scaled to the different measuring times. The $\mu^{+}$ data were taken at reduced spectrometer field. 
The probability $A_{\mu e}$ for a $\mu^{-} \rightarrow \mathrm{e}^{-}$conversion event to be selected in the final sample has been determined by simulation, and whenever possible from the experimental data themselves. $A_{\mu e}$ accounts for the geometric acceptance and the efficiencies of the trigger, the event reconstruction and selection cuts. As a preliminary result we find $A_{\mu e} \approx 10 \%$. Combined with the total number of muon stops (Eq. 4.11) and the probability of nuclear capture $(0.972(3)$ [6] corresponds to a single-event sensitivity of $2-3 \times 10^{-13}$. The final result for the upper limit on the branching ratio should become available before the end of the year.

\section{References}

[1] R. Barbieri, L. Hall and A. Strumia, 'iNucl. Phys. B $445(1995) 21 \overline{1}$.

[2] T.S. Kosmas, Inv. talk at NuFACT'01 Workshop, May 24-30, 2001, Tsukuba, Ibaraki, Japan, to appear in Nucl. Instr. Meth..

T.S. Kosmas and I.E. Lagaris, to be submitted to Nucl. Phys. A.

T.S. Kosmas, Z. Ren and A. Faessler, 'Nucul. Phys. A 665. (2000) 183i, and references therein.

[3] W. Honecker et al., 'Phys. Rev. Lett. $76 \mathbf{6}(1996) 200$ '

M.Grossmann-Handschin et al., iNucl. Instrum.

[4] R. Watanabe et al., Atomic Data and Nucl. Data Tables 54 (1993) 165.

[5] F.J. Hartmann et al., Zeits. f. Physik A 305 (1982) 189.

[6] T. Suzuki et al., 'Phys. 